\title{
Selecting the most suitable next-generation in-building network: from copper-based to optical solutions
}

\author{
Bart Lannoo, Koen Casier, Mathias Gheeraert, Jan Van Ooteghem, Sofie Verbrugge, Didier Colle, \\ Mario Pickavet, Piet Demeester \\ Dep. of Information Technology, Ghent University - IBBT, Gaston Crommenlaan 8, B-9050 Gent, Belgium \\ Tel: (32) 9 14998, Fax: (32) 9 14899,e-mail: bart.lannoo@intec.ugent.be
}

\begin{abstract}
Next generation optical access networks will increase the bandwidth to the customer substantially and even beyond the current in-building network bandwidth. The same way optical is revolutionizing the access network, there is an ongoing trend where optical is used for in-building networks as well. Different optical solutions are available today, each with their specific application areas. Often it is not only a choice between different optical transmission media, but also the topology and installation method can differ according to the situation. Next to the new optical solutions, copper-based technologies can still have several advantages in new installations. Choices are based on the installation economics and several influencing parameters like building status, type and size, available infrastructure and bandwidth requirements. This paper presents a detailed techno-economic model for all different fixed in-building network solutions and this model is applied in various realistic scenarios. As a conclusion, the paper also presents a general selection scheme for different in-building scenarios, based on the technical requirements and the outcome of the techno-economic calculations. This scheme can be used as a general guideline for choosing the most suitable in-building network solution in a specific scenario.
\end{abstract}

Keywords: In-building networks, Cat, POF, MMF, SMF, Techno-economics,

\section{INTRODUCTION}

With the advent of fibre-to-the-home (FTTH), the access network bandwidth offered is typically $100 \mathrm{Mbps}$ and can in the future be easily upgraded to more than $1 \mathrm{Gbps}$. The current in-building networks, if existing, typically provide up to $100 \mathrm{Mbps}$ and cannot easily be upgraded to carry more. Wireless technologies provide a much easier installation, but its bandwidth is often insufficient as it diminishes fast with an increasing number of users (due to its shared nature) and when crossing walls and windows. Installation of new fixed in-building network ducting and cabling requires substantial work. In extension to existing papers [1], this paper makes both a quantitative comparison of the different existing solutions as well as a qualitative selection. The first makes a comparison between several technologies based on the estimated installation cost versus the bandwidth attainable. The second can be used to detect the best candidates for a given scenario. Finally, the results of this research also link to previous techno-economic studies on the installation cost of an FTTH network and show how customers can take full advantage of the offered high bandwidth.

\section{GENERAL STRUCTURE OF AN IN-BUILDING NETWORK}

An in-building network covers the whole indoor telecommunication infrastructure and equipment between the residential gateway (RG) and the end user. The RG is installed where the network enters the building and it corresponds to the demarcation point between the access and the in-building network. The end point of the network can consist of different terminals like PCs, TVs, printers, etc. Between both, an in-building network infrastructure is deployed, e.g. as depicted in Fig. 1. We recognize the following network components:

- Cabling: the physical medium (copper or fibre), used for the data-transport.

- Ducting: the infrastructure in which the cables are deployed; they can be installed on the walls or in a buried manner.

- Connector: the connection between the cable segments and other network components.

- Wall outlet: the end points of the network; where user access is given to the telecommunication network.

In addition, following components can be part of the network, depending on the used transmission medium and the applied network configuration:

- Switch: device that takes care of the communication between the different network segments of a shared medium and the RG. A switch forwards incoming data packets to the addressed ports (i.e. a packetswitched connection).

- Hub: just like a switch, a hub allows communication between the different network segments and the RG. In contrast to a switch, a hub sends the incoming data packets simply to all ports in het network.

- Media convertor: a device that makes the optical/electrical (O/E) conversion in case of fibre cabling, and it is an important component in fibre-based in-building networks. 


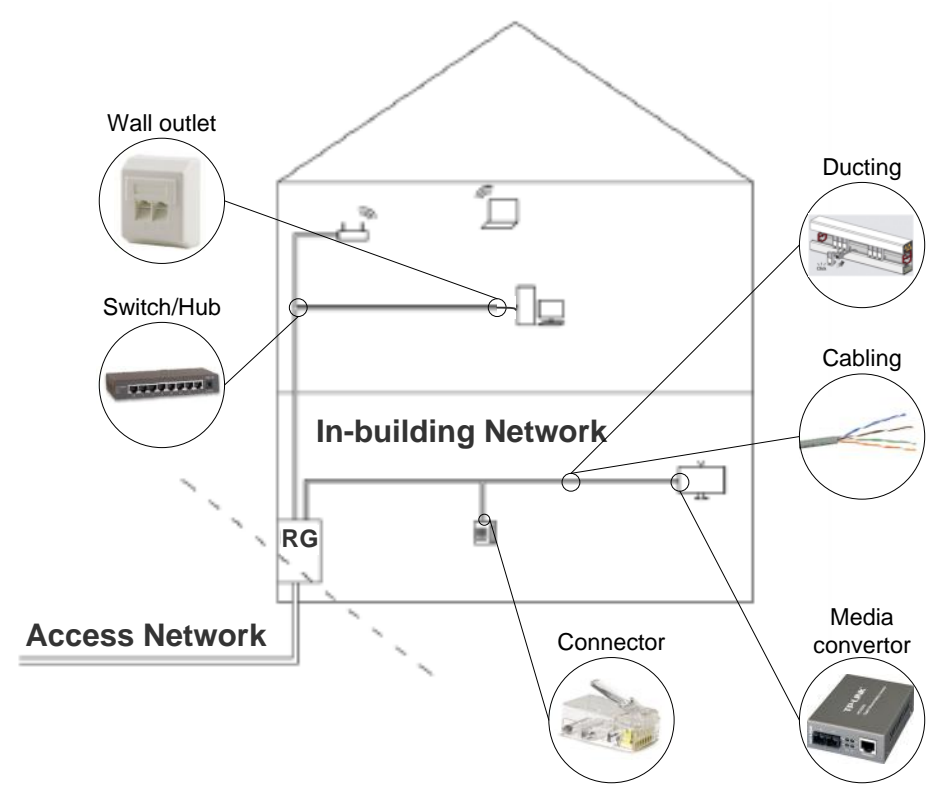

Figure 1: Main component blocks of an in-building network

\section{COST VS. BANDWIDTH IN THE INSTALLATION OF AN IN-BUILDING NETWORK}

We have built a full techno-economic model for estimating the costs of installing a new in-building network infrastructure depending on the following parameters: number of building floors, rooms per floor, room dimensions and brown-field vs. green-field installations. We adapted the model to work with the installation and intermediate conversion of formats (e.g., optical to copper based) for the following five types of fixed cabling technologies: Ethernet Cat5e and Cat6A, plastic optical fibre (POF), silica multimode fibre (MMF) and silica single-mode fibre (SMF). The cost breakdown for a new point to point (P2P) installation in a building with three floors and four apartments on each floor is shown in Fig. 2 (left) for an installation in 2011. Clearly the prices of all equipment rapidly decrease in time and this will especially be the case for optical equipment which does not yet have the same mature market as Ethernet has nowadays. We used an extended learning curve to estimate the decrease of the price of all types of media convertors in time as defined in [2]. The results of such decrease in price over a time of ten years are shown in Fig. 2 (right). By that time POF has the lowest cost and SMF and MMF are only slightly more expensive than Ethernet. In the case of SMF and MMF, the differences become smaller, making the choice for SMF at that point in time more justified. In the case the market would select one of the new types as a standard for higher bandwidth transport, then clearly the situation would more rapidly evolve, and media convertors for this type can be integrated in end-user equipment (e.g., PC, TV), making it the installation of choice.
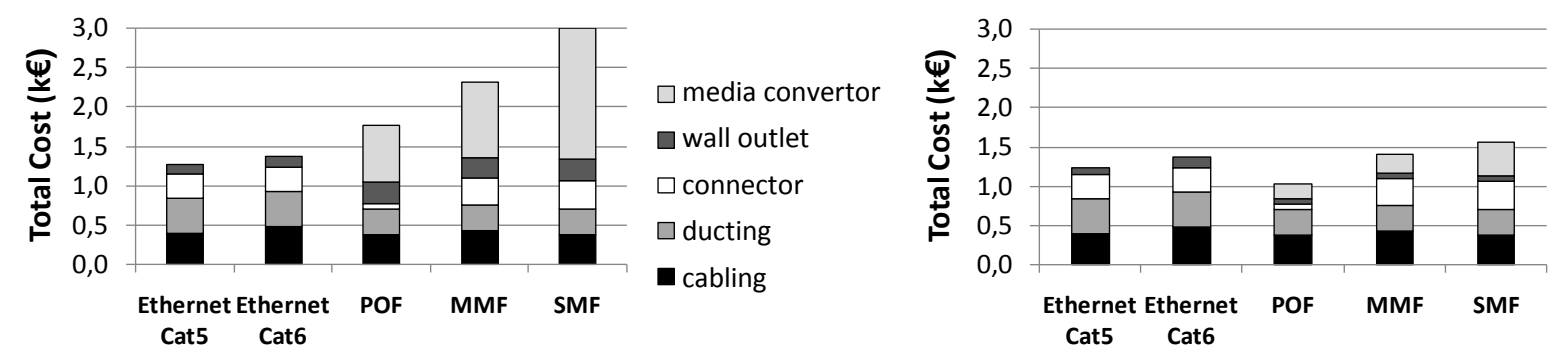

Figure 2: Breakdown of the costs for an in-building P2P installation in 2011 (left) and in 2020 (right)

Next we take a closer look at the bandwidth in relation to the cost of the different solutions. Fig. 3 (left) shows the cost of the installation of an MMF solution as a function of the size of a building in terms of the number of apartments per floor (for a building with three floors). The costs of a P2P installation rapidly increase for an increase in the number of rooms per floor and for more than four rooms per floor, a bus topology per floor becomes the cheaper solution. In order to find the cost in relation to the bandwidth, we have to take into account the bandwidth decrease for a bus installation. While it is clear that we would choose a bus topology for more 
than five rooms per floor, the bandwidth of a bus topology with $N$ rooms in a row leads to a decrease of the dedicated bandwidth with a factor $N$.

Connecting to a next generation optical network infrastructure, it is fair to consider an in-building dedicated network requirement of at least $100 \mathrm{Mbps}$, which also corresponds to the commonly used Fast Ethernet standard. We consider the maximum attainable bandwidth of Cat5e to be $100 \mathrm{Mbps}$; of Cat6A, POF and MMF to be $1 \mathrm{Gbps}$, and of SMF much more than $1 \mathrm{Gbps}$. These bandwidths, however, can be considered as current reference values, but might increase in the future thanks to new technical evolutions. Fig. 3 (right) shows the cost of the lowest cost solution, switching from P2P to bus and back to P2P for all considered technologies whenever the bandwidth of the bus topology offers less than $100 \mathrm{Mbps}$ dedicated. The figure shows that the cost of Cat5e is higher than Cat6A when a Cat6A bus topology is possible. The figure also shows that currently POF is for larger installations, due to the lower cabling cost comparable to Cat6A. In the short term future clearly the cost of optical in-building networks might well become lower than that of Ethernet equipment, especially when the media convertors become cheaper or obsolete.
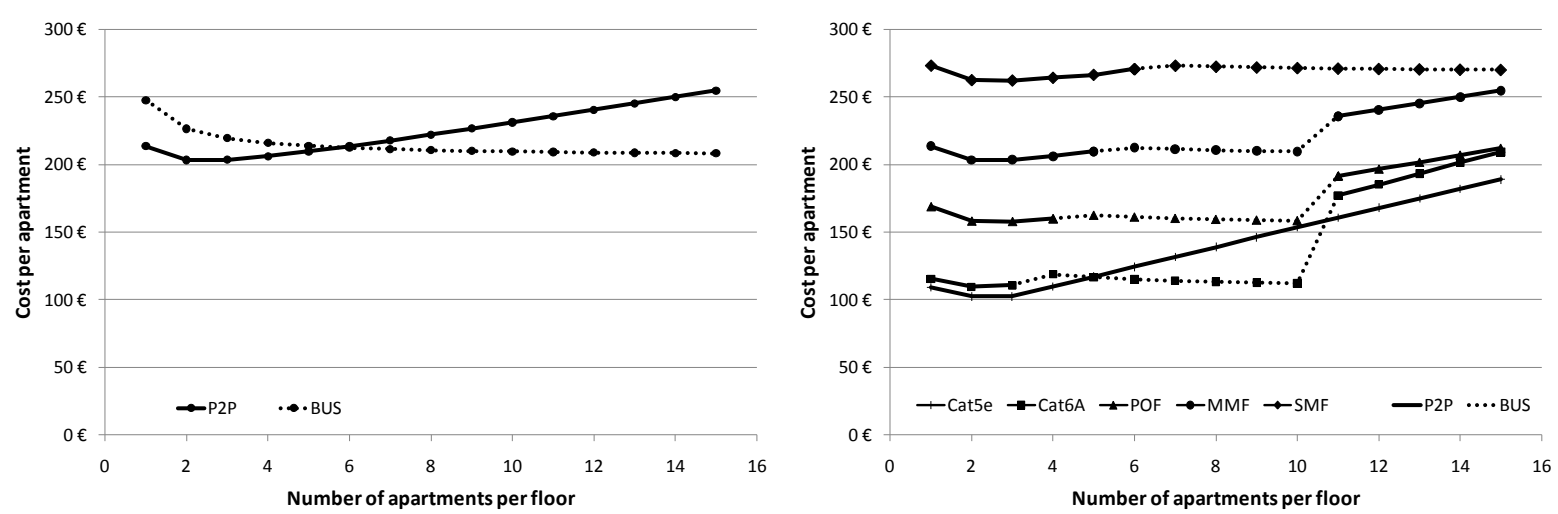

Figure 3: Installation cost in function of the number of rooms per floor for MMF (left) and for five technologies taking cost and bandwidth demand into account (right)

\section{SELECTING THE MOST SUITED IN-BUILDING NETWORK}

The main reason for upgrading the in-building network, especially in case of high-rise buildings, is the shortage of bandwidth in the connection to the access network. Clearly when upgrading, the installation should be made as future proof as possible. In practice this means that the dedicated bandwidth should be sufficient for future applications and the peak bandwidth should be able to follow future evolutions of the access network. Fig. 4 depicts a flow chart to indicate the optimal choices for the installation of new in-building network infrastructure, taking into account the results from the previous section.

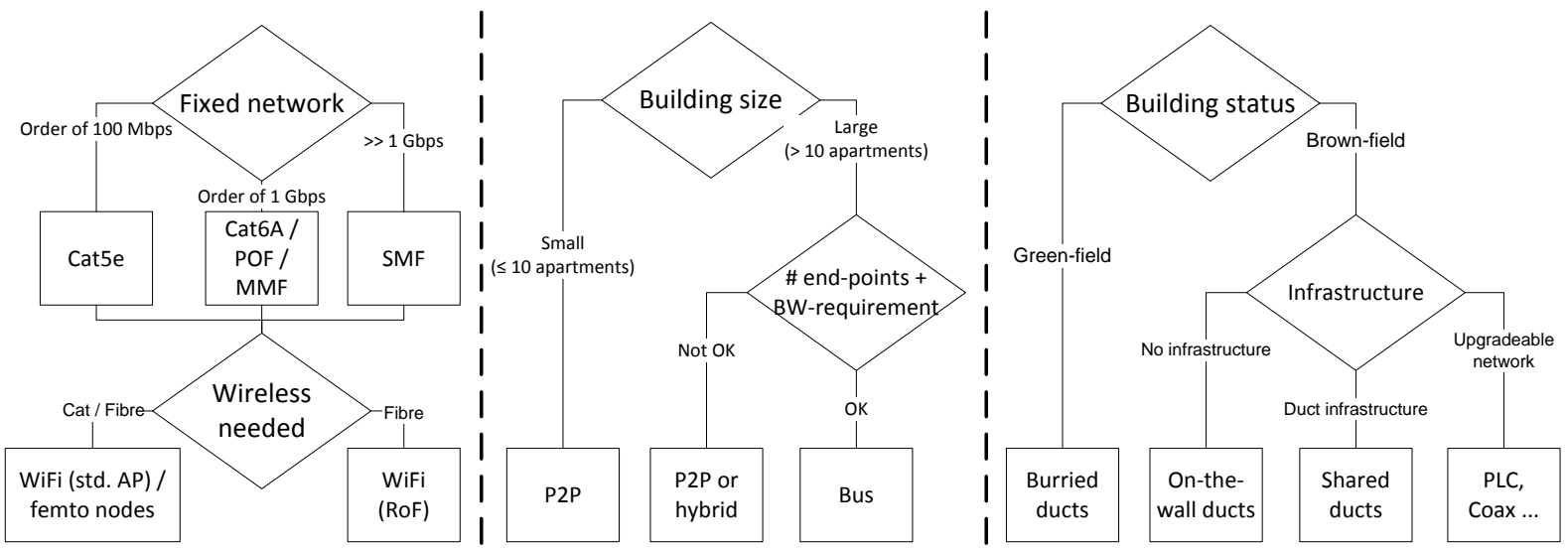

Figure 4: Flow chart to indicate the optimal in-building network installation

A first parameter that will be of importance for the selection of the in-building network will of course be the bandwidth aimed at for the installed network. In an installation with low constraints and little to no challenging applications the customers can choose for a Cat5e solution. This will offer the cheapest solution as Ethernet is a well known and on massive scale produced solution. In smaller installations, e.g. residential houses, Cat5e could 
even provide the possibility to offer up to $1 \mathrm{Gbps}$. Taking a look at the future and especially when looking at larger installations, e.g. high rise buildings, choosing for either a very easy to (later) install plastic optical fibre (POF) solution or for a silica optical fibre - easiest with multimode fibre (MMF), or more future proof with single-mode fibre (SMF) - would provide the best solution. In the different cases a wireless network can be attached to the fixed network, albeit in the case of an optical fibre solution the wireless signal can be generated at a central location and sent over the network up to the antenna in a so-called radio over fibre (RoF) solution. This will in large buildings reduce the installation costs of wireless antennas in each apartment of the building. In SMF and MMF, this is possible in the base frequency of the wireless signal. In POF, a conversion to the right carrier frequency will be required or over longer distance typically the users will have to work with standard access points.

The second parameter of importance is the building size. It is important to make a distinction between larger (high rise) buildings, e.g., with more than ten apartments, and lower buildings. This will especially be important for the selection of the best installation approach, as illustrated in Fig. 3 where the tradeoffs between bandwidth, costs and installation size were evaluated. In a smaller building, the distances to the different apartments will be small and in this case, a P2P installation can be cost effective and will also provide the highest dedicated bandwidth, most flexibility and the best future proof solution, even with a Cat5e solution. In the case of a high rise building, it is more cost effective to work with a bus or hybrid solution in which each floor is connected on a bus and with a P2P from each floor to the access network connection. The selection between both should be made based on the expected bandwidth and the amount of end points to be connected. Here, the technology of choice will have an important impact as well, as an SMF cable offers more bandwidth than an MMF or POF solution. In very large cases or office buildings, this could lead to choose an SMF installation as preferred technology.

The third parameter of importance is the building status. When installing an in-building network infrastructure, it makes a lot of difference if the installation is done in a new building (green-field) or in an existing one (brownfield). In case of a green-field installation, it is straightforward to work with buried ducts as this is the best protected and most esthetical solution. For existing buildings, however, burying the ducts is very labour intensive and on-the-wall mounted ducts offer a valuable alternative in case a complete new infrastructure is needed. Alternatively, in some buildings an existing network infrastructure can be reused for providing broadband access, either an existing duct infrastructure (e.g., sharing the ducts used for the electricity network) or an upgradeable network (e.g., the electricity network by using power line communication or PLC, the existing coax network by using multimedia over coax alliance or MoCA). Although the latter solutions are sometimes less future-proof, they can provide a very cost-effective alternative that meets the current requirements.

\section{CONCLUSION}

When fibre access networks come to the home and to the building, high bandwidth connections are enabled up to the customer equipment. Many new applications can make use of the higher bandwidth. Still this is only the truth in case the in-building network has a comparable high bandwidth from the point where the access line comes in the rooms or apartments of the building. Currently, this is often not the case, especially not when access bandwidth would still increase, and an upgrade of the in-building network is required to accommodate the current and future bandwidth demands. We presented some results from a techno-economic model which allows estimating the costs for the installation of a new in-building network infrastructure in detail. The results clearly show the trade-off between the building size, customer bandwidth and costs and indicate which installation approach to use in which case. It also shows that optical in-building networks are currently more expensive in installation than Ethernet equipment, but might in the short term future become cheaper than Ethernet especially when the media convertors become cheaper or optical interfaces are integrated in the end-user equipment. Based on the techno-economic results, this paper also presented a selection scheme which shows the best candidate technology and installation approach - P2P, bus or hybrid - when given a particular situation in terms of bandwidth, building size and number of end points.

\section{ACKNOWLEDGEMENT}

This research was carried out as part of the IBBT TERRAIN project. This project is co-funded by IBBT, IWT and Acreo AB, Alcatel-Lucent, Comsof, Deutsche Telekom Laboratories, Digipolis, FTTH Council Europe, Geosparc, Stad Gent, TMVW, TE Connectivity, UNET and WCS Benelux BV.

\section{REFERENCES}

[1] A.M.J. Koonen, H.P.A. van den Boom, E. Tangdiongga, H.-D. Jung, P. Guignard, “Designing in-building optical fiber networks", Proc. of OFC 2010, San Diego, California, USA, Mar. 21-25, 2010, paper JThA46.

[2] B. T. Olsen and K. Stordahl, "Models for forecasting cost evolution of components and technologies", Telektronikk 4.04, pp. 138-148, 2004. 